\title{
Consistency and Asymptotic Normality of the Maximum Likelihood Estimator in GaGLM
}

\author{
Benchao Wang, Hong Gu, and Pan Qin
}

\begin{abstract}
The Gamma distribution based generalized linear model (GaGLM) is a kind of statistical model feasible for the positive value of a non-stationary stochastic system, in which the location and the scale are regressed by the corresponding explanatory variables. This paper theoretically investigates the asymptotic properties for maximum likelihood estimates (MLE) of $G a \mathrm{GLM}$, which can benefit the further interval estimates, hypothesis tests and stochastic control design. First, the score function and the Fisher information matrix for GaGLM are derived. Then, the Lyapunov condition is derived to ensure the asymptotic normality of the score function normalized by the Fisher information matrix. Based on this condition, the asymptotic normality of the MLE of GaGLM is proven. Finally, a numerical example is given to testify the asymptotic properties obtained in the research. The numerical results indicate that the MLE of GaGLM converged to a normal distribution as the number of sample measurements increased.
\end{abstract}

\section{Index Terms} mator.

Asymptotic Theory, Gamma distribution, Gamma regression, Generalized linear model, maximum likelihood esti-

\section{INTRODUCTION}

$\mathbf{T}$ HE generalized linear model (GLM) expands the general linear model so that a dependent variable is linearly related to the factors and covariates via a specified link function [1]. Moreover, the model allows the dependent variable to keep the attribute of actually applied data, such as integer literal, positive and asymmetric, not belong to a normal distribution. It covers widely used statistical models, such as logistic regression models for binary distributed responses, Poisson regression models for count data and Gamma regression models for positive real data.

As a family of moderate skewness and continuous phenomena distributions, the Gamma distribution is a useful model in many areas of statistics when the normal distribution is not appropriate. In the Gamma distribution-based approach, the system output $Z$ can be assumed to be a subject $Z \sim G a(\alpha, \beta)$, where $G a(\alpha, \beta)$ is a Gamma distribution with the shape parameter $\alpha$ and the rate parameter $\beta$ governing its probability density function shape. This distribution was first introduced [2] and subsequently studied in detail [3]. In some special cases, the Gamma distribution reduces to the exponential distribution as $\alpha=1$ and $\beta=1 / \lambda$, the Erlang distribution as $\alpha=n$ and the $\chi^{2}$ distribution as $\alpha=n / 2$ and $\beta=1 / 2$.

Because of the flexibility of the relationship to many other distributions, the Gamma distribution can be a suitable alternative for modelling such kinds of the positive-valued dependent variable. The Gamma distribution-based models have been applied in many areas, such as medical science [4], [5], biology [6], economics [7], [8], forest science [9] and education [10]. Considering the ubiquitous heteroscedasticity of actually applied data, as a member of the well-known GLM, the Gamma distribution based generalized linear model ( $G a \mathrm{GLM}$ ) is more widely used when $\alpha$ and $\beta$ (or $\mu$ and $k$ ) are both dependent variables. However, it should be noted that the GaGLM does not belong to the exponential family of distributions based GLM. Therefore, it is necessary to establish a baseable asymptotic theory for $G a$ GLM.

This research investigates the theoretical aspects of maximum likelihood estimator (MLE) for GaGLM. Because $G a$ GLM is a model with two equations being respectively parameterized for $\alpha$ and $\beta$, the estimation procedure could be relatively complex. In statistics, several expectation-maximization (EM) type algorithms have been developed for the Gamma distribution inference, where $\beta$ was assumed to be a latent variable [11]. However, those algorithms were developed by fixing $\beta$ as constant. If $\beta$ parameterized as regression models, the EM algorithm would be extremely computationally involved. Thus, rather than using the EM algorithm, we directly solve MLE for GaGLM by using the Fisher scoring algorithm [12]. To this end, the score function and the Fisher information matrix are derived for $G a$ GLM. Furthermore, we obtain the condition to assure the positive definiteness of the Fisher information matrix.

The consistency and the asymptotic normality explaining the efficiency of the estimators have been widely investigated in system identification and statistics [13], [14]. The consistency of MLE for GaGLM can be proved by using the same approach for GLM [14]. To verify the asymptotic normality of MLE, the asymptotic normality of the normalized

B. Wang is with Faculty of Electronic Information and Electrical Engineering, Dalian University of Technology, Dalian, 116024, China, and also with Department of Administration and Supervision, Liaoning Police College, Dalian, 116036, China(e-mail: wangbc@mail.dlut.edu.cn).

H. Gu and P. Qin are with Faculty of Electronic Information and Electrical Engineering, Dalian University of Technology, Dalian, 116024, China(e-mail: guhong@dlut.edu.cn; qp112cn@dlut.edu.cn). 
score function is necessary. GLM was developed for the exponential family, whose moment generating functions are exponential functions of the sufficient statistics. Based on the uniform moment generating function, the asymptotic normality of the normalized score function was proved for GLM.

To investigate asymptotic properties of MLE for parameters occurring in GaGLM, we need prove the consistency and asymptotic normality of MLE by central limit theorems. Compared with commonly used Lindeberg condition, Lyapunov condition is stronger in proving asymptotic properties. First, we derived the score functions normalized by the Fisher information matrix for the Lyapunov condition, which ensure the asymptotic normality of the normalized score functions [15]. Based on this result, the asymptotic normality of MLE for GaGLM is finally proved. These results can dramatically facilitate the hypothesis testing, the construction of interval estimates, and stochastic control design for the non-stationary stochastic system [16], [17].

The rest of this paper is organized as follows. The concept of GaGLM and maximum likelihood estimation are introduced in Section II. Section III gives the assumptions of asymptotic properties of the MLE in GaGLM, including the proof of related lemmas and theorems. Results of a simulation study are reported in Section IV. Concluding comments are presented in Section V.

\section{Problem Statement}

In this section, we briefly review GaGLM, including its structure and numerical method of MLE.

\section{A. Model and estimation}

Suppose that we observe realizations of a positive real random variable $Z$, and we believe that $Z$ has a specified positive continuous distribution.

Let $D_{n}=\left\{\left(Z_{i}, \boldsymbol{x}_{i}, \boldsymbol{y}_{i}\right), i=1, \ldots, n\right\}$ be independent random vectors defined on the probability space $(\Omega, \mathcal{F}, \mathbb{P})$. For each $i=1, \ldots, n$, the response variable $Z_{i}$ is generated from the following process:

$$
Z_{i} \sim G a\left(\alpha_{i}, \beta_{i}\right),
$$

where $G a\left(\alpha_{i}, \beta_{i}\right)$ denotes the Gamma distribution with positive shape parameter $\alpha_{i}$ and rate parameter $\beta_{i}$. The probability density function is

$$
f\left(Z_{i} \mid \alpha_{i}, \beta_{i}\right)= \begin{cases}\frac{\beta_{i}^{\alpha_{i}}}{\Gamma\left(\alpha_{i}\right)} z_{i}^{\alpha_{i}-1} e^{-\beta_{i} z_{i}}, & \text { when } z>0 \\ 0, & \text { when } z \leq 0,\end{cases}
$$

where $\Gamma(\cdot)$ is the Gamma function. The mean and variance of the random variable $Z_{i}$ are given by

$$
E\left(Z_{i}\right)=\frac{\alpha_{i}}{\beta_{i}}
$$

and

$$
\operatorname{Var}\left(Z_{i}\right)=\frac{\alpha_{i}}{\beta_{i}^{2}}
$$

Then, we can develop GaGLM by regressing explainatory variable $\boldsymbol{\omega}_{i}=\left(\boldsymbol{x}_{i}^{\top}, \boldsymbol{y}_{i}^{\top}\right)^{\top}$ with $\boldsymbol{x}_{i} \in \mathbb{R}^{p}$ to $\alpha_{i}$ and $\boldsymbol{y}_{i} \in \mathbb{R}^{q}$ to $\beta_{i}$ as follows:

$$
\left\{\begin{array}{l}
\alpha_{i}=\exp \left(\boldsymbol{x}_{i}^{\top} \boldsymbol{a}\right) \\
\beta_{i}=\exp \left(\boldsymbol{y}_{i}^{\top} \boldsymbol{b}\right)
\end{array}\right.
$$

where $\boldsymbol{a}=\left(a_{1}, \ldots, a_{p}\right)$ and $\boldsymbol{b}=\left(b_{1}, \ldots, b_{q}\right)$ denote the regression parameter vectors for $\alpha_{i}$ and $\beta_{i}$ respectively, and $\bullet^{\top}$ denotes the transpose of $\bullet$. Further, $\boldsymbol{\theta}=\left(\boldsymbol{a}^{\top}, \boldsymbol{b}^{\top}\right)^{\top}$ is any parameter in an admissible set $K_{\boldsymbol{\theta}} \subset \mathbb{R}^{p+q}$. For the observations $z_{1}, z_{2}, \ldots, z_{n}$, the log-likelihood $l(\boldsymbol{\theta})$ derived from the GaGLM can be written as

$$
\begin{aligned}
l_{n}(\boldsymbol{\theta}) & =\sum_{i=1}^{n} f\left(Z_{i} \mid \boldsymbol{\omega}_{i}, \boldsymbol{\theta}\right) \\
& =\sum_{i=1}^{n}\left(\alpha_{i} \log \beta_{i}-\log \Gamma\left(\alpha_{i}\right)+\alpha_{i} \log z_{i}-\log z_{i}-\beta_{i} z_{i}\right) \\
& =\sum_{i=1}^{N}\left(e^{\boldsymbol{x}_{i}^{\top} \boldsymbol{a}} \boldsymbol{y}_{i}^{\top} \boldsymbol{b}-\log \Gamma\left(e^{\boldsymbol{x}_{i}^{\top} \boldsymbol{a}}\right)+e^{\boldsymbol{x}_{i}^{\top} \boldsymbol{a}} \log z_{i}-\log z_{i}-e^{\boldsymbol{y}_{i}^{\top} \boldsymbol{b}} z_{i}\right)
\end{aligned}
$$


Then, $\boldsymbol{\theta}$ can be estimated by

$$
\widehat{\boldsymbol{\theta}}=\arg \max l_{n}(\boldsymbol{\theta}) .
$$

According to (5), the first three order derivative of $l_{n}(\boldsymbol{\theta})$ with respect to $\boldsymbol{\theta}$ is continuous and finite for all $\boldsymbol{\theta} \in K_{\boldsymbol{\theta}}$. This condition ensures the existence of the Taylor expansion, the finite variance of the derivatives of $l_{n}(\boldsymbol{\theta})$. Thus, MLE can be obtained by the scoring method [19], in which the score function can be obtained by

$$
\begin{aligned}
\boldsymbol{s}_{n}(\boldsymbol{\theta}) & =\frac{\partial l_{n}(\boldsymbol{\theta})}{\partial \boldsymbol{\theta}} \\
& =\left(\boldsymbol{s}_{\boldsymbol{a}}^{\top}(\boldsymbol{\theta}), \boldsymbol{s}_{\boldsymbol{b}}^{\top}(\boldsymbol{\theta})\right)^{\top} \\
& =\left[\begin{array}{ll}
\frac{\partial l_{n}(\boldsymbol{\theta})}{\partial \boldsymbol{a}^{\top}} & \frac{\partial l_{n}(\boldsymbol{\theta})}{\partial \boldsymbol{b}^{\top}}
\end{array}\right]^{\top},
\end{aligned}
$$

and the Fisher information matrix can be obtained by

$$
\begin{aligned}
\boldsymbol{F}_{n}(\boldsymbol{\theta}) & =E\left[\frac{\partial l_{n}(\boldsymbol{\theta})}{\partial \boldsymbol{\theta}} \frac{\partial l_{n}(\boldsymbol{\theta})}{\partial \boldsymbol{\theta}^{\top}}\right] \\
& =\left(\begin{array}{ll}
E\left(\frac{\partial l_{n}(\boldsymbol{\theta})}{\partial \boldsymbol{a}} \frac{\partial l_{n}(\boldsymbol{\theta})}{\partial \boldsymbol{a}^{\top}}\right) & E\left(\frac{\partial l_{n}(\boldsymbol{\theta})}{\partial \boldsymbol{a}} \frac{\partial l_{n}(\boldsymbol{\theta})}{\partial \boldsymbol{b}^{\top}}\right) \\
E\left(\frac{\partial l_{n}(\boldsymbol{\theta})}{\partial \boldsymbol{b}} \frac{\partial l_{n}(\boldsymbol{\theta})}{\partial \boldsymbol{a}^{\top}}\right) & E\left(\frac{\partial l_{n}(\boldsymbol{\theta})}{\partial \boldsymbol{b}} \frac{\partial l_{n}(\boldsymbol{\theta})}{\partial \boldsymbol{b}^{\top}}\right)
\end{array}\right) .
\end{aligned}
$$

With the score function and the Fisher information matrix, (6) can be iteratively solved by using the generalized Newton-Raphson (NR) method, so-called Fisher's scoring (FS) algorithm [19] as the following

$$
\left.\widehat{\boldsymbol{\theta}}_{(n e w)}=\widehat{\boldsymbol{\theta}}_{(\text {old })}+\boldsymbol{F}_{n}^{-1} \widehat{\boldsymbol{\theta}}_{(\text {old })}\right) \boldsymbol{s}_{n}\left(\widehat{\boldsymbol{\theta}}_{(\text {old })}\right) .
$$

In what follows, the score function and the Fisher information matrix are derived for GaGLM. Furthermore, the condition that ensures the positive definiteness of $\boldsymbol{F}_{n}(\boldsymbol{\theta})$ obtained in Corollary 1.

In statistics, the asymptotic properties, mainly including the consistency and asymptotic normality, are often used to evaluate the efficiency of estimators [20]. Another important role of $\boldsymbol{s}_{n}(\boldsymbol{\theta})$ and $\boldsymbol{F}_{n}(\boldsymbol{\theta})$ is to prove the asymptotic properties. If the first three order derivates of $l_{n}(\boldsymbol{\theta})$ with respect of $\boldsymbol{\theta}$ exist, the consistency, i.e. $\widehat{\boldsymbol{\theta}}$ converging in probability to the true coefficients $\boldsymbol{\theta}_{0}$, can be proved under a generalized framework [21]. However, the asymptotic converged of the covariance matrix for GaGLM cannot be proved by using the generalized approach in [21]. To tackle this problem, we first prove the asymptotic normality of the normalized score function $\boldsymbol{F}_{n}^{-\mathrm{T} / 2}(\boldsymbol{\theta}) \boldsymbol{s}_{n}(\boldsymbol{\theta})$ motivated by [14]. Note that [14] dealt with the exponential family-based models, whose moment generating function is the exponential function of the sufficient statistics. [14] used such moment generating function to prove the asymptotic normality of the normalized score function. However, there is not an asymptotic theory of MLE to Gamma distribution, where the approach in [14] cannot be extended to the GaGLM. Furthermore, the elements constructing $\boldsymbol{F}_{n}^{-\mathrm{T} / 2}(\boldsymbol{\theta}) \boldsymbol{s}_{n}(\boldsymbol{\theta})$ cannot be expected to be identically distributed. Thus, we investigate the Lyapunov condition and the Taylor expansion, the asymptotic normality of MLE of GaGLM can be proved. In what follows, we first derive the score function and the Fisher information matrix of MLE of GaGLM.

\section{Score Function And Fisher information matrix for GaGLM}

For deriving the score function and the Fisher information matrix, the log-likelihood function of $\boldsymbol{\theta}$ is formulated from (6). The score function (7) can be represented as follows.

Lemma 1: (Component-wise score function for $G a \mathrm{GLM})$. The two components of the score function (7) are obtained by

$$
\begin{aligned}
\boldsymbol{s}_{\boldsymbol{a}}(\boldsymbol{\theta}) & =\frac{\partial l_{n}(\boldsymbol{\theta})}{\partial \boldsymbol{a}} \\
& =\left(s_{1}(\boldsymbol{\theta}), \ldots, s_{p}(\boldsymbol{\theta})\right)^{\top} \\
& =\sum_{i=1}^{n}\left(\log \beta_{i}-\psi_{0}\left(\alpha_{i}\right)+\log z_{i}\right) \\
& =\sum_{i=1}^{n}\left(\alpha_{i} \log \beta_{i}-\psi_{0}\left(\alpha_{i}\right) \alpha_{i}+\alpha_{i} \log z_{i}\right) \boldsymbol{x}_{i}
\end{aligned}
$$


and

$$
\boldsymbol{s}_{\boldsymbol{b}}(\boldsymbol{\theta})=\frac{\partial l_{n}(\boldsymbol{\theta})}{\partial \boldsymbol{b}}=\sum_{i=1}^{n}\left(\alpha_{i}-\beta_{i} z_{i}\right) \boldsymbol{y}_{i}
$$

where $\psi_{0}(\alpha)=\frac{\Gamma^{\prime}(\alpha)}{\Gamma(\alpha)}$ is digamma function, which can be seen in Equation (19).

Proof Lemma 1: The following derivatives can be directly derived from (4).

$$
\left\{\begin{array}{l}
\frac{\partial \alpha_{i}}{\partial \boldsymbol{a}}=\alpha_{i} \boldsymbol{x}_{i} \\
\frac{\partial \beta_{i}}{\partial \boldsymbol{b}}=\beta_{i} \boldsymbol{y}_{i} .
\end{array}\right.
$$

Then, derivatives of the log-likelihood function (5) are straightforwardly obtained.

The Fisher information matrix will be derived via the Hessian matrix follows

$$
\begin{aligned}
\mathcal{H}_{n}(\boldsymbol{\theta}) & =\left[\begin{array}{ll}
\frac{\partial l_{n}(\boldsymbol{\theta})}{\partial \boldsymbol{a}} \frac{\partial l_{n}(\boldsymbol{\theta})}{\partial \boldsymbol{a}^{\top}} & \frac{\partial l_{n}(\boldsymbol{\theta})}{\partial \boldsymbol{a}} \frac{\partial l_{n}(\boldsymbol{\theta})}{\partial \boldsymbol{b}^{\top}} \\
\frac{\partial l_{n}(\boldsymbol{\theta})}{\partial \boldsymbol{b}} \frac{\partial l_{n}(\boldsymbol{\theta})}{\partial \boldsymbol{a}^{\top}} & \frac{\partial l_{n}(\boldsymbol{\theta})}{\partial \boldsymbol{b}} \frac{\partial l_{n}(\boldsymbol{\theta})}{\partial \boldsymbol{b}^{\top}}
\end{array}\right] \\
: & =\left[\begin{array}{ll}
\boldsymbol{h}_{p, p}(\boldsymbol{\theta}) & \boldsymbol{h}_{p, q}(\boldsymbol{\theta}) \\
\boldsymbol{h}_{q, p}(\boldsymbol{\theta}) & \boldsymbol{h}_{q, q}(\boldsymbol{\theta})
\end{array}\right],
\end{aligned}
$$

with entries:

$$
\begin{aligned}
\boldsymbol{h}_{p, p}(\boldsymbol{\theta}) & =\frac{\partial^{2} l_{n}(\theta)}{\partial \boldsymbol{a} \partial \boldsymbol{a}^{\boldsymbol{\top}}} \\
& =\sum_{i=1}^{n} \alpha_{i}\left[\log \beta_{i}+\log z_{i}-\psi_{0}\left(\alpha_{i}\right)\right. \\
& \left.-\alpha_{i} \psi_{1}\left(\alpha_{i}\right)\right] \boldsymbol{x}_{i} \boldsymbol{x}_{i}^{\top}
\end{aligned}
$$

where $\psi_{1}(\alpha)=\psi^{\prime}(\alpha)$ is trigamma function, which can be seen in Equation (20).

$$
\begin{aligned}
\boldsymbol{h}_{q, q}(\boldsymbol{\theta}) & =\frac{\partial^{2} l_{n}(\theta)}{\partial \boldsymbol{b} \partial \boldsymbol{b}^{\boldsymbol{\top}}} \\
& =-\sum_{i=1}^{n} \beta_{i} z_{i} \boldsymbol{y}_{i} \boldsymbol{y}_{i}^{\boldsymbol{\top}}
\end{aligned}
$$

and

$$
\begin{aligned}
\boldsymbol{h}_{p, q}(\boldsymbol{\theta})=\boldsymbol{h}_{q, p}^{\top}(\boldsymbol{\theta}) & =\frac{\partial^{2} l_{n}(\theta)}{\partial \boldsymbol{a} \partial \boldsymbol{b}^{\top}} \\
& =\sum_{i=1}^{n} \alpha_{i} \boldsymbol{x}_{i} \boldsymbol{y}_{i}^{\top}
\end{aligned}
$$

In order to derive the Fisher information matrix and prove asymptotic normality, the following Lemmas 2 and 3 are necessary.

Lemma 2: If $Z \sim G a(\alpha, \beta)$ then

$$
E(\log Z)=\psi_{0}(\alpha)-\log \beta
$$

and

$$
E(\log Z)^{k} \leq C(\alpha, \beta, k), \quad k=1,2, \ldots, n
$$

where $C$ is positive constant depending on $\alpha, \beta$ and $k$, and $k>0$ is any finite positive integer. 
Proof Lemma 2: Before the proof of this lemma, we should recall the Euler's gamma function $\Gamma(\alpha)$ and digamma function $\psi_{0}(\alpha)$ for $\alpha>0$ defined as

$$
\psi_{0}(\alpha)=\frac{\Gamma^{\prime}(\alpha)}{\Gamma(\alpha)}, \quad \text { with } \Gamma(\alpha)=\int_{0}^{\infty} u^{\alpha-1} e^{-u} d u
$$

For basic properties of these functions see [22]. Polygamma functions $\psi_{n}$, such as trigamma, tetragamma and pentagamma functions when $n=1,2,3$, are defined to be $n$-order derivatives of $\psi_{0}$ function, that is,

$$
\psi_{n}(\alpha)=\psi_{0}^{(n)}(\alpha), \quad n=1,2, \ldots
$$

The following integral and series representations are valid for $z>0$ and $n=1,2,3, \ldots$ :

$$
\begin{aligned}
(-1)^{n-1} \psi_{n}(\alpha) & =\int_{0}^{\infty} \frac{t^{n} e^{-\alpha t}}{1-e^{-t}} d t \\
& =n ! \sum_{k=0}^{\infty} \frac{1}{(\alpha+k)^{n+1}} \quad(\alpha>0),
\end{aligned}
$$

which are monotonically increasing and continuous function in $\alpha>0$ [23]. Then, We can get $\frac{\Gamma^{(k)}(\alpha)}{\Gamma(\alpha)}$ by polygamma functions with $k=1,2, \ldots, n$. When $n=1$, we can get

$$
\begin{aligned}
\psi_{1}(\alpha) & =\psi_{0}^{\prime}(\alpha) \\
& =\left(\frac{\Gamma^{\prime}(\alpha)}{\Gamma(\alpha)}\right)^{\prime} \\
& =\frac{\Gamma^{\prime \prime}(\alpha)}{\Gamma(\alpha)}-\left(\frac{\Gamma^{\prime}(\alpha)}{\Gamma(\alpha)}\right)^{2} \\
& =\frac{\Gamma^{\prime \prime}(\alpha)}{\Gamma(\alpha)}-\psi_{0}^{2}(\alpha),
\end{aligned}
$$

then

$$
\frac{\Gamma^{\prime \prime}(\alpha)}{\Gamma(\alpha)}=\psi_{1}(\alpha)+\psi_{0}^{2}(\alpha)
$$

When $n=2$, we can get

$$
\begin{aligned}
\psi_{2}(\alpha) & =\psi_{1}^{\prime}(\alpha) \\
& =\left(\frac{\Gamma^{\prime \prime}(\alpha)}{\Gamma(\alpha)}\right)^{\prime}-\left(\psi_{0}^{2}(\alpha)\right)^{\prime} \\
& =\frac{\Gamma^{\prime \prime \prime}(\alpha)}{\Gamma(\alpha)}-\frac{\Gamma^{\prime \prime}(\alpha) \Gamma^{\prime}(\alpha)}{\Gamma^{2}(\alpha)}-2 \psi_{0}(\alpha) \psi^{\prime}(\alpha) \\
& =\frac{\Gamma^{\prime \prime \prime}(\alpha)}{\Gamma(\alpha)}-\left(\psi_{1}(\alpha)+\psi_{0}^{2}(\alpha)\right) \psi_{0}(\alpha)-2 \psi_{0}(\alpha) \psi_{1}(\alpha) \\
& =\frac{\Gamma^{\prime \prime \prime}(\alpha)}{\Gamma(\alpha)}-\psi_{0}^{3}(\alpha)-3 \psi_{0}(\alpha) \psi_{1}(\alpha) .
\end{aligned}
$$

In the following,

$$
\frac{\Gamma^{\prime \prime \prime}(\alpha)}{\Gamma(\alpha)}=\psi_{2}(\alpha)+\psi_{0}^{3}(\alpha)+3 \psi_{0}(\alpha) \psi_{1}(\alpha)
$$

Continuous derivative of (19) function as $(22)$ and $(23)$, we can get $\frac{\Gamma^{(k)}(\alpha)}{\Gamma(\alpha)}$ function.

$$
\frac{\Gamma^{(k)}(\alpha)}{\Gamma(\alpha)}=f_{k \Gamma}\left(\psi_{0}(\alpha), \psi_{1}(\alpha), \psi_{2}(\alpha), \ldots, \psi_{k-1}(\alpha)\right),
$$

where $f_{k \Gamma}\left(\psi_{0}(\alpha), \psi_{1}(\alpha), \psi_{2}(\alpha), \ldots, \psi_{k-1}(\alpha)\right)$ is a finite j-order polynomial combination function of the $\psi_{i}(\alpha)$ functions with $i<k$ and $j \leq k$.

Let $Z \sim G a(\alpha, \beta)$, we have 


$$
\int_{0}^{\infty} \frac{1}{\Gamma(\alpha)} \beta^{\alpha} z^{\alpha-1} e^{-\beta z} d z=1
$$

Multiply at each side of equation (25) by $\Gamma(\alpha)$, we can get

$$
\Gamma(\alpha)=\int_{0}^{\infty} \beta^{\alpha} z^{\alpha-1} e^{-\beta z} d z
$$

Then, take the $k$-order derivative with respect to $\alpha$ of both sides, that

$$
\Gamma^{(k)}(\alpha)=\sum_{i=0}^{k} C_{k}^{i} \int_{0}^{\infty} \beta^{\alpha} z^{\alpha-1} e^{-\beta z} \log ^{k-i} \beta \log ^{i} z d z .
$$

Divided by $\Gamma(\alpha)$ at both sides of $(27)$, we obtain

$$
\begin{aligned}
\frac{\Gamma^{(k)}(\alpha)}{\Gamma(\alpha)} & =\sum_{i=0}^{k} C_{k}^{i} \int_{0}^{\infty} \frac{1}{\Gamma(\alpha)} \beta^{\alpha} z^{\alpha-1} e^{-\beta z} \log ^{k-i} \beta \log ^{i} z d z \\
& =\sum_{i=0}^{k} C_{k}^{i} \log ^{k-i} \beta E(\log Z)^{i} .
\end{aligned}
$$

For $k=1$,

$$
\begin{aligned}
\psi_{0}(\alpha) & =\int_{0}^{\infty} \frac{1}{\Gamma(\alpha)} \beta^{\alpha} \log \beta z^{\alpha-1} e^{-\beta z} d z+\int_{0}^{\infty} \frac{1}{\Gamma(\alpha)} \beta^{\alpha} z^{\alpha-1} \log z e^{-\beta z} d z \\
& =\log \beta+E(\log Z)
\end{aligned}
$$

then

$$
E(\log Z)=\psi_{0}(\alpha)-\log \beta .
$$

Let $f_{k}(\bullet)$ denote finite $j$-order polynomial of $\log \beta$ by $j \leq k$ and linear combination of $E(\log Z)^{i}$ with $i=1,2, \ldots, k-1$ function. Combining (24) and (26), we can get

$$
\begin{aligned}
E(\log Z)^{k} & =\frac{\Gamma^{(k)}(\alpha)}{\Gamma(\alpha)}+f_{k}\left(\log \beta, E(\log Z), E(\log Z)^{2}, \ldots, E(\log Z)^{k-1}\right) \\
& =f_{k}\left(\log \beta, E(\log Z), E(\log Z)^{2}, \ldots, E(\log Z)^{k-1}\right)+f_{k \Gamma}\left(\psi_{0}(\alpha), \psi_{1}(\alpha), \psi_{2}(\alpha), \ldots, \psi_{k-1}(\alpha)\right) \\
& \leq C(\alpha, \beta, k)
\end{aligned}
$$

Lemma 3: If $Z \sim G a(\alpha, \beta)$, the $k$ th moment of $\mathrm{Z}$ is limited as

$$
E Z^{k} \leq C(\alpha, \beta, k), \quad k=1,2, \ldots, n
$$

where $C$ is positive constant depending on $\alpha, \beta$ and $k$, and $k>0$ is any finite positive integer.

Proof Lemma 3:

$$
\begin{aligned}
E Z^{k} & =\int_{0}^{\infty} \frac{1}{\Gamma(\alpha)} \beta^{\alpha} z^{k+\alpha-1} e^{-\beta z} d z \\
& =\int_{0}^{\infty} \frac{\Gamma(k+\alpha)}{\Gamma(\alpha)} \frac{\beta^{-k}}{\Gamma(k+\alpha)} \beta^{k+\alpha} z^{k+\alpha-1} e^{-\beta z} d z \\
& =\frac{\Gamma(k+\alpha) \beta^{-k}}{\Gamma(\alpha)} \int_{0}^{\infty} \frac{1}{\Gamma(k+\alpha)} \beta^{k+\alpha} z^{k+\alpha-1} e^{-\beta z} d z \\
& =\frac{\Gamma(k+\alpha) \beta^{-k}}{\Gamma(\alpha)} \\
& \leq C(\alpha, \beta, k)
\end{aligned}
$$


Theorem 1: (Fisher information matrix for GaGLM). The components of the Fisher information matrix are obtained as follows:

$$
\begin{gathered}
E\left(\frac{\partial l_{n}(\boldsymbol{\theta})}{\partial \boldsymbol{a}} \frac{\partial l_{n}(\boldsymbol{\theta})}{\partial \boldsymbol{a}^{\top}}\right)=\sum_{i=1}^{n}\left(\alpha_{i} \log \beta_{i}-\psi_{0}\left(\alpha_{i}\right) \alpha_{i}+\alpha_{i} \log z_{i}\right) \boldsymbol{x}_{i} \boldsymbol{x}_{i}^{\top} \\
E\left(\frac{\partial l_{n}(\boldsymbol{\theta})}{\partial \boldsymbol{b}} \frac{\partial l_{n}(\boldsymbol{\theta})}{\partial \boldsymbol{b}^{\top}}\right)=\sum_{i=1}^{n} \alpha_{i} \boldsymbol{y}_{i} \boldsymbol{y}_{i}^{\top} \\
E\left(\frac{\partial l_{n}(\boldsymbol{\theta})}{\partial \boldsymbol{a}} \frac{\partial l_{n}(\boldsymbol{\theta})}{\partial \boldsymbol{b}^{\top}}\right)=-\sum_{i=1}^{n} \alpha_{i} \boldsymbol{x}_{i} \boldsymbol{y}_{i}^{\top}
\end{gathered}
$$

Proof Theorem 1: Under the assumptions of mild general regularity, we have $\boldsymbol{F}_{n}(\boldsymbol{\theta})=-E \mathcal{H}_{n}(\boldsymbol{\theta})$ by $[24]$, and $\boldsymbol{F}_{n}(\boldsymbol{\theta})$ is positive semi-definite matrix [25]. Thus, using Lemmas 1, 2, equations (8) and (13), the Fisher information matrix can be straightforward computed as follows

$$
\begin{aligned}
\boldsymbol{F}_{n}(\boldsymbol{\theta}) & =c-E \mathcal{H}_{n}(\boldsymbol{\theta}) \\
& =-\left[\begin{array}{ll}
E \boldsymbol{h}_{p, p}(\boldsymbol{\theta}) & E \boldsymbol{h}_{p, q}(\boldsymbol{\theta}) \\
E \boldsymbol{h}_{q, p}(\boldsymbol{\theta}) & E \boldsymbol{h}_{q, q}(\boldsymbol{\theta})
\end{array}\right] \\
& =\left[\begin{array}{ll}
\boldsymbol{f}_{p, p}(\boldsymbol{\theta}) & \boldsymbol{f}_{p, q}(\boldsymbol{\theta}) \\
\boldsymbol{f}_{q, p}(\boldsymbol{\theta}) & \boldsymbol{f}_{q, q}(\boldsymbol{\theta})
\end{array}\right] .
\end{aligned}
$$

According to (17) in lemma 2, they are expressed respectively as follows

$$
\begin{aligned}
& \boldsymbol{f}_{p, p}=-E\left[\boldsymbol{h}_{p, p}(\boldsymbol{\theta})\right] \\
&=-E\left[\sum_{i=1}^{n} \alpha_{i}\left(\log \beta_{i}+\log z_{i}-\psi_{0}\left(\alpha_{i}\right)-\alpha_{i} \psi_{1}\left(\alpha_{i}\right)\right) \boldsymbol{x}_{i} \boldsymbol{x}_{i}^{\top}\right] \\
&=\sum_{i=1}^{n} \alpha_{i}^{2} \psi_{1}\left(\alpha_{i}\right) \boldsymbol{x}_{i} \boldsymbol{x}_{i}^{\top} \\
& \boldsymbol{f}_{q, q}=-E\left[\boldsymbol{h}_{q, q}(\boldsymbol{\theta})\right] \\
&=-E \sum_{i=1}^{n} \beta_{i} z_{i} \boldsymbol{y}_{i} \boldsymbol{y}_{i}^{\top} \\
&=\sum_{i=1}^{n} \alpha_{i} \boldsymbol{y}_{i} \boldsymbol{y}_{i}^{\top}
\end{aligned}
$$

and

$$
\begin{aligned}
\boldsymbol{f}_{p, q}(\boldsymbol{\theta})=\boldsymbol{f}_{q, p}^{\top}(\boldsymbol{\theta}) & =-E\left[\boldsymbol{h}_{p, q}(\boldsymbol{\theta})\right] \\
& =-E \sum_{i=1}^{n} \alpha_{i} \boldsymbol{x}_{i} \boldsymbol{y}_{i}^{\top} \\
& =-\sum_{i=1}^{n} \alpha_{i} \boldsymbol{x}_{i} \boldsymbol{y}_{i}^{\top} .
\end{aligned}
$$

Then, we can get

$$
\begin{aligned}
\boldsymbol{F}_{n}(\boldsymbol{\theta}) & =\sum_{i=1}^{n}\left[\begin{array}{cc}
\alpha_{i}^{2} \psi_{1}\left(\alpha_{i}\right) & -\alpha_{i} \\
-\alpha_{i} & \alpha_{i}
\end{array}\right]\left[\begin{array}{ll}
\boldsymbol{x}_{i} \boldsymbol{x}_{i}^{\top} & \boldsymbol{x}_{i} \boldsymbol{y}_{i}^{\top} \\
\boldsymbol{y}_{i} \boldsymbol{x}_{i}^{\top} & \boldsymbol{y}_{i} \boldsymbol{y}_{i}^{\top}
\end{array}\right] \\
& =\sum_{i=1}^{n} \alpha_{i}\left[\begin{array}{cc}
\alpha_{i} \psi_{1}\left(\alpha_{i}\right) & -1 \\
-1 & 1
\end{array}\right]\left[\begin{array}{l}
\boldsymbol{x}_{i} \\
\boldsymbol{y}_{i}
\end{array}\right]\left[\begin{array}{ll}
\boldsymbol{x}_{i}^{\top} & \boldsymbol{y}_{i}^{\top}
\end{array}\right]
\end{aligned}
$$

Corollary 1: (Definiteness of Fisher information matrix for GaGLM). If $\sum_{i=1}^{n} \boldsymbol{\omega}_{i} \boldsymbol{\omega}_{i}^{\top}$ is of full rank, with $\boldsymbol{\omega}_{i}=$ $\left(\boldsymbol{x}_{i}^{\top}, \boldsymbol{y}_{i}^{\top}\right)^{\top}$ denoted in Section II, the Fisher information matrix $\boldsymbol{F}_{n}(\boldsymbol{\theta})$ is positive-definite. 
Proof Corollary 1: To prove the positive character of the Fisher information matrix, we need derive the range of $\alpha \psi_{1}(\alpha)$. From the equation (21) as $n=1$, we can get following inequality

$$
\begin{aligned}
\psi_{1}(\alpha) & =\sum_{k=0}^{\infty} \frac{1}{(\alpha+k)^{2}} \\
& >\sum_{k=0}^{\infty} \frac{1}{(\alpha+k)(\alpha+k+1)} \\
& =\sum_{k=0}^{\infty}\left(\frac{1}{\alpha+k}-\frac{1}{\alpha+k+1}\right) \\
& =\frac{1}{\alpha}-\frac{1}{\alpha+1}+\frac{1}{\alpha+1}-\frac{1}{\alpha+2}+\frac{1}{\alpha+2}-\ldots \\
& =\frac{1}{\alpha} .
\end{aligned}
$$

Then, we can get

$$
\alpha_{i}\left|\begin{array}{cc}
\alpha_{i} \psi_{1}\left(\alpha_{i}\right) & -1 \\
-1 & 1
\end{array}\right|=\alpha_{i}\left(\alpha_{i} \psi_{1}\left(\alpha_{i}\right)-1\right)>0
$$

If $\sum_{i=1}^{n} \boldsymbol{\omega}_{i} \boldsymbol{\omega}_{i}^{\top}$ is of full rank, the Fisher information matrix $\boldsymbol{F}_{n}(\boldsymbol{\theta})$ is positive-definite.

\section{Asymptotic theory for the maximum Likelihood estimator in GaGLM}

Under the mild assumptions, the asymptotic properties of the MLE was proved in GLM for canonical link functions [14]. These asymptotic conditions can be applied to prove similar results for GaGLM as well as noncanonical.

To normalize the score function, we introduce the Cholesky square root matrix for positive definite matrix $\boldsymbol{F}$, such that $\boldsymbol{F}^{1 / 2}\left(\boldsymbol{F}^{1 / 2}\right)^{\top}=\boldsymbol{F}$. We set $\boldsymbol{F}^{1 / 2}$ denotes the unique lower triangular matrix with positive diagonal elements. For convenience, set $\boldsymbol{F}^{\mathrm{T} / 2}:=\left(\boldsymbol{F}^{1 / 2}\right)^{\top}, \boldsymbol{F}^{-1 / 2}:=\left(\boldsymbol{F}^{1 / 2}\right)^{-1}$ and $\boldsymbol{F}^{-\mathrm{T} / 2}:=\left(\boldsymbol{F}^{\mathrm{T} / 2}\right)^{-1}$. For convenience, we drop the argument $\boldsymbol{\theta}_{0}$ in $\boldsymbol{s}_{n}\left(\boldsymbol{\theta}_{0}\right), \boldsymbol{s}_{n i}\left(\boldsymbol{\theta}_{0}\right), \boldsymbol{F}_{n}\left(\boldsymbol{\theta}_{0}\right) E_{\boldsymbol{\theta}_{0}}$ etc. and write $\boldsymbol{s}_{n}, \boldsymbol{s}_{n i}, \boldsymbol{F}_{n}, E$ etc. $C_{i}$ for $i=1,2, \ldots$ will further denote constants, with or without subindexes. The same $C$ 's represent different constants in different formula.

Let $\|\cdot\|$ denote the spectral norm of square matrices. The spectral norm of a real-valued matrix $\boldsymbol{F}$ is given by

$$
\begin{aligned}
\|\boldsymbol{F}\| & =\left(\lambda_{\max }\left(\boldsymbol{F} \boldsymbol{F}^{\boldsymbol{\top}}\right)\right)^{1 / 2} \\
& =\sup _{\|\boldsymbol{u}\|_{2}=1}\|\boldsymbol{F} \boldsymbol{u}\|_{2},
\end{aligned}
$$

where $\|\cdot\|_{2}$ denotes the $L^{2}$ - norm of vectors. The maximal (minimal) eigenvalue of a square matrix $\boldsymbol{F}$ will be further denoted by $\lambda_{\max }(\boldsymbol{F})\left(\lambda_{\min }(\boldsymbol{F})\right)$. For $\varepsilon>0$, a neighborhood of the unknown true parameter $\boldsymbol{\theta}_{0}$ can denote by

$$
N_{n}(\varepsilon)=\left\{\boldsymbol{\theta}:\left\|\boldsymbol{F}_{n}^{\boldsymbol{\top} / 2} \cdot\left(\boldsymbol{\theta}-\boldsymbol{\theta}_{0}\right)\right\| \leq \varepsilon\right\} .
$$

In this paper, let's make the following assumptions.

(A1)

$$
\lambda_{\min }\left(\boldsymbol{F}_{n}\right) \geq \frac{n}{C} \quad \forall n \geq 1,
$$

where $C$ is a positive constant.

(A2) $\left\{\boldsymbol{x}_{n}, n \geq 1\right\} \subset K_{x},\left\{\boldsymbol{y}_{n}, n \geq 1\right\} \subset K_{y}$, where $K_{x} \subset \mathbb{R}^{p}$ and $K_{y} \subset \mathbb{R}^{q}$ are compact sets.

(A3) $K_{\boldsymbol{\theta}} \subset \mathbb{R}^{p+q}$ is an open set, and $\boldsymbol{\theta}_{0}$ is an interior point of the set $K_{\boldsymbol{\theta}}$.

Furthermore, Assumption (A1) means that $\lambda_{\min }\left(\boldsymbol{F}_{n}\right)$ and $n$ are the same order infinity, which is used to prove Lemmas 4 and 5. Assumption (A2) implies what we deal with are compact regressors. If $\boldsymbol{\theta}$ lies on the boundary of parameter space $K_{\boldsymbol{\theta}}$, the statements of Theorem 2 do not valid anymore.

Based on the assumptions above, we need to prove two preliminary Lemmas 4 and 5 for asymptotic properties of MLE $\widehat{\boldsymbol{\theta}}$ first. 
Lemma 4: Under the assumptions (A1)-(A3), there is

$$
\boldsymbol{F}_{n}^{-\mathrm{T} / 2} \boldsymbol{s}_{n} \stackrel{D}{\longrightarrow} N_{P}\left(\mathbf{0}, \boldsymbol{I}_{p+q}\right) \quad \text { as } n \rightarrow \infty
$$

where $N_{P}\left(\mathbf{0}, \boldsymbol{I}_{p+q}\right)$ is a $(p+q)$-dimensional normal distribution with mean vector $\mathbf{0}$ and covariance matrix $\boldsymbol{I}_{p+q}$.

Proof Lemma 4: Derived from Cramer-Wald [20], we only need to prove that a linear combination $\boldsymbol{u}^{\top} \boldsymbol{F}_{n}^{-1 / 2} \boldsymbol{s}_{n}$ converges in distribution to $N\left(0, \boldsymbol{u}^{\top} \boldsymbol{u}\right)$ for any vector $\boldsymbol{u} \in \mathbb{R}^{p+q}(\boldsymbol{u} \neq \mathbf{0})$. Without loss of generality, we set $\|\boldsymbol{u}\|=1$. Then, let

$$
\begin{aligned}
\boldsymbol{s}_{n}(\boldsymbol{\theta}) & =\left(\boldsymbol{s}_{\boldsymbol{a}}^{\top}(\boldsymbol{\theta}), \boldsymbol{s}_{\boldsymbol{b}}^{\top}(\boldsymbol{\theta})\right)^{\top} \\
& =\left(s_{1}(\boldsymbol{\theta}), \ldots, s_{p}(\boldsymbol{\theta}), s_{p+1}(\boldsymbol{\theta}), \ldots, s_{p+q}(\boldsymbol{\theta})\right)^{\top}
\end{aligned}
$$

where

$$
s_{r}(\boldsymbol{\theta}):=\frac{\partial l_{n}(\boldsymbol{\theta})}{\partial a_{r}}=\sum_{i=1}^{n} s_{r, i}(\boldsymbol{\theta})
$$

with

$$
s_{r, i}(\boldsymbol{\theta})=\left(\alpha_{i} \log \beta_{i}-\psi_{0}\left(\alpha_{i}\right) \alpha_{i}+\alpha_{i} \log z_{i}\right) x_{i r}
$$

for $r=1, \ldots, p$, and $\psi_{0}(\cdot)$ is digamma function (seen in Equation (19)).

$$
s_{p+r}(\boldsymbol{\theta}):=\frac{\partial l_{n}(\boldsymbol{\theta})}{\partial b_{r}}=\sum_{i=1}^{n} s_{p+r, i}(\boldsymbol{\theta})
$$

with

$$
s_{p+r, i}(\boldsymbol{\theta})=\left(\alpha_{i}-\beta_{i} z_{i}\right) y_{i r}
$$

for $r=1, \ldots, q$.

Now observe that $\boldsymbol{s}_{n}$ can be written as a sum of independent random vectors, namely

$$
\boldsymbol{s}_{n}=\sum_{i=1}^{n} \boldsymbol{s}_{n i}
$$

where $\boldsymbol{s}_{n i}=\left(s_{1, i}, \ldots, s_{p, i}, s_{p+1, i}, \ldots, s_{p+q, i}\right)^{\top}$ with $s_{r, i}:=s_{r, i}\left(\boldsymbol{\theta}_{0}\right)$ defined in $(48)$ and $(49)$ for $r=1,2, \ldots, p+q$ and $i=$ $1, \ldots, n$, respectively. Further, define independent random variables $\xi_{\text {in }}$ by $\xi_{\text {in }}:=\boldsymbol{u}^{\top} \boldsymbol{F}_{n}^{-1 / 2} \boldsymbol{s}_{n i}$. Since $E\left(\xi_{\text {in }}\right)=0$ and $\operatorname{Var}\left(\sum_{i=1}^{n} \xi_{i n}\right)=1$, it is enough to show that the Lyapunov condition is satisfied, i.e.

$$
L_{s}:=\sum_{i=1}^{n} E\left|\xi_{\text {in }}\right|^{s} \stackrel{n \rightarrow \infty}{\longrightarrow} 0, \quad \exists s>2 .
$$

Let $s=3$ (see p. 393, e.g., Hoffmann [26]). Noticing that $\left\|\boldsymbol{F}_{n}^{-1 / 2}\right\|^{2}=1 / \lambda_{\min }(\boldsymbol{F})$, it follows from (A1) that

$$
\begin{aligned}
L_{3} & \leq \sum_{i=1}^{n} E\left(\left\|\boldsymbol{u}^{\top}\right\|^{3}\left\|\boldsymbol{F}_{n}^{-1 / 2}\right\|^{3}\left\|\boldsymbol{s}_{n i}\right\|^{3}\right) \\
& \leq \frac{C}{n^{3 / 2}} \sum_{i=1}^{n} E\left\|\boldsymbol{s}_{n i}\right\|^{3} \\
& \leq \frac{C}{\sqrt{n}} \max _{i=1, \ldots, n} E\left\|\boldsymbol{s}_{n i}\right\|^{3} .
\end{aligned}
$$

Using an extension of the $c_{r}$-inequality given by

$$
E\left|\sum_{i=1}^{n} \zeta_{i}\right|^{k} \leq n^{k-1} \sum_{i=1}^{n} E\left|\zeta_{i}\right|^{k} \quad(k>1, k \in \mathbb{R}),
$$


to $n$ arbitrary random variables $\zeta_{1}, \ldots, \zeta_{n}$ (see p.58, e.g., Petrov [27]) yields that

$$
\begin{aligned}
E\left\|\boldsymbol{s}_{n i}\right\|^{3} & \leq(p+q)^{2}\left(E\left|s_{1, i}\right|^{3}+E\left|s_{2, i}\right|^{3}+\ldots+E\left|s_{p+q, i}\right|^{3}\right) \\
& \leq C\left(E\left|s_{1, i}\right|^{3}+E\left|s_{2, i}\right|^{3}+\ldots+E\left|s_{p+q, i}\right|^{3}\right) .
\end{aligned}
$$

Thus, it remains to establish that $\max _{i=1, \ldots, n} E\left|s_{p, i}\right|$ is uniformly bounded in $n$ for $r=1, \ldots, p+q$. This will be shown for case $r=1, \ldots, p$ and $r=p+1, \ldots, p+q$. The remaining cases can be treated similarly. Without loss of generality, set $r=p$ and $r=p+q$ respectively. Using Lemma 2 and formula (53), we have

$$
\begin{aligned}
& \max _{i=1, \ldots, n} E\left|s_{p, i}\right|^{3} \\
= & \max _{i=1, \ldots, n} E\left|x_{i p}\left(\alpha_{i} \log \beta_{i}-\psi_{0}\left(\alpha_{i}\right) \alpha_{i}+\alpha_{i} \log Z_{i}\right)\right|^{3} \\
= & \max _{i=1, \ldots, n} E\left(\left|x_{i p}\right|^{3}\left|\alpha_{i} \log \beta_{i}-\psi_{0}\left(\alpha_{i}\right) \alpha_{i}+\alpha_{i} \log Z_{i}\right|^{3}\right) \\
\leq & C \max _{\boldsymbol{x} \in K_{x}, \boldsymbol{y} \in K_{y}}\|\boldsymbol{x}\|^{3}\left[E\left|\exp \left(\boldsymbol{x}^{\top} \boldsymbol{a}\right) \boldsymbol{y}^{\top} \boldsymbol{b}\right|^{3}+E\left|\psi_{0}\left(\exp \left(\boldsymbol{x}^{\top} \boldsymbol{a}\right)\right) \exp \left(\boldsymbol{x}^{\top} \boldsymbol{a}\right)\right|^{3}+E\left|\exp \left(\boldsymbol{x}^{\top} \boldsymbol{a}\right) \log Z_{i}\right|^{3}\right] \\
\leq & C_{1}\left(\boldsymbol{\theta}_{0}\right)+C_{2}\left(\boldsymbol{\theta}_{0}\right) \max _{\boldsymbol{x} \in K_{x}, \boldsymbol{y} \in K_{y}} E\left|\log Z_{i}\right|^{3} \\
\leq & C_{1}\left(\boldsymbol{\theta}_{0}\right)+C_{2}\left(\boldsymbol{\theta}_{0}\right) \max _{\boldsymbol{x} \in K_{x}, \boldsymbol{y} \in K_{y}} \sqrt{E\left(\log Z_{i}\right)^{6}} \\
\leq & C_{3}\left(\boldsymbol{\theta}_{0}\right),
\end{aligned}
$$

where $Z_{i} \sim G a\left(\alpha_{i}, \beta_{i}\right)$ for $i=1, \ldots, n$ and $Z \sim G a\left(\exp \left(\boldsymbol{x}^{\top} \boldsymbol{a}\right), \exp \left(\boldsymbol{y}^{\top} \boldsymbol{b}\right)\right)$, and

$$
\begin{aligned}
& \max _{i=1, \ldots, n} E\left|s_{p+q, i}\right|^{3} \\
= & \max _{i=1, \ldots, n} E\left(y_{i q}\left(\alpha_{i}-\beta_{i} Z_{i}\right)\right) \\
\leq & \max _{i=1, \ldots, n} E\left(\left|y_{i q}\right|^{3} \cdot\left|\alpha_{i}-\beta_{i} Z_{i}\right|^{3}\right) \\
\leq & \max _{\boldsymbol{x} \in K_{x}, \boldsymbol{y} \in K_{y}}\|\boldsymbol{y}\|^{3} E\left|\exp \left(\boldsymbol{x}_{i}^{\top} \boldsymbol{a}\right)-\exp \left(\boldsymbol{y}_{i}^{\top} \boldsymbol{b}\right) Z_{i}\right|^{3} \\
\leq & C_{\boldsymbol{x} \in K_{x}, \boldsymbol{y} \in K_{y}}\|\boldsymbol{y}\|^{3}\left(E\left|\exp \left(\boldsymbol{x}_{i}^{\top} \boldsymbol{a}\right)\right|^{3}+E\left|\exp \left(\boldsymbol{y}_{i}^{\top} \boldsymbol{b}\right) Z_{i}\right|^{3}\right) \\
\leq & C_{1}\left(\boldsymbol{\theta}_{0}\right)+C_{2}\left(\boldsymbol{\theta}_{0}\right) \max _{\boldsymbol{x} \in K_{x}, \boldsymbol{y} \in K_{y}} E Y_{i}^{3} \\
\leq & C_{3}\left(\boldsymbol{\theta}_{0}\right) .
\end{aligned}
$$

We can get

$$
L_{3} \stackrel{n \rightarrow \infty}{\longrightarrow} 0
$$

then

$$
\boldsymbol{F}_{n}^{-\mathrm{T} / 2} \boldsymbol{s}_{n} \stackrel{D}{\rightarrow} N_{P}\left(\mathbf{0}, \boldsymbol{I}_{p+q}\right)
$$

Lemma 5: Under the assumptions (A1)-(A3)

$$
\max _{\theta \in N_{n}(\varepsilon)}\left\|\boldsymbol{V}_{n}(\boldsymbol{\theta})-\boldsymbol{I}_{p+q}\right\| \stackrel{P}{\rightarrow} 0, \quad \forall \varepsilon>0,
$$

where $\boldsymbol{V}_{n}(\boldsymbol{\theta}):=\boldsymbol{F}_{n}^{-1 / 2} \boldsymbol{H}_{n}(\boldsymbol{\theta}) \boldsymbol{F}_{n}^{-\mathrm{T} / 2}$ for $n=1,2, \ldots$

Proof Lemma 5: We have a.s.

$$
\begin{aligned}
\left\|\boldsymbol{V}_{n}(\boldsymbol{\theta})-\boldsymbol{I}_{p+q}\right\| & =\left\|\boldsymbol{F}_{n}^{-1 / 2}\left(\boldsymbol{H}_{n}(\boldsymbol{\theta})-\boldsymbol{F}_{n}\right) \boldsymbol{F}_{n}^{-\mathrm{T} / 2}\right\| \\
& \leq \frac{1}{\lambda_{\min }\left(\boldsymbol{F}_{n}\right)}\left\|\boldsymbol{H}_{n}(\boldsymbol{\theta})-\boldsymbol{F}_{n}\right\| \\
& \leq \frac{C}{n}\left\|\boldsymbol{H}_{n}(\boldsymbol{\theta})-\boldsymbol{F}_{n}\right\|
\end{aligned}
$$

Thus, conditions 


$$
\max _{\theta \in N_{n}(\varepsilon)}\left\|\frac{1}{n}\left(\boldsymbol{H}_{n}(\boldsymbol{\theta})-E \boldsymbol{H}_{n}(\boldsymbol{\theta})\right)\right\| \stackrel{p}{\rightarrow} 0
$$

and

$$
\max _{\theta \in N_{n}(\varepsilon)}\left\|\frac{1}{n}\left(E \boldsymbol{H}_{n}(\boldsymbol{\theta})-\boldsymbol{F}_{n}\right)\right\| \stackrel{p}{\rightarrow} 0
$$

imply (59).

To prove (61), it is sufficient to establish that the $(j, k)$-element of the random matrix $\left(\boldsymbol{H}_{n}(\boldsymbol{\theta})-E \boldsymbol{H}_{n}(\boldsymbol{\theta})\right) / n$ converges to zero in probability, i.e.

$$
\max _{\theta \in N_{n}(\varepsilon)}\left|\frac{h_{j, k}(\boldsymbol{\theta})-E h_{j, k}(\boldsymbol{\theta})}{n}\right| \stackrel{p}{\rightarrow} 0
$$

There are three different types of entries (14), (15) and (16) in the Hessian matrix. We will show the convergence of formula $(63)$ in the cases of $1 \leq j, k \leq p$. It is similar to treat the remaining cases. In order to avoid generality, let $j=p$ and $k=p$, then

$$
\max _{\theta \in N_{n}(\varepsilon)} \frac{1}{n}\left|h_{p, p}(\boldsymbol{\theta})-E h_{p, p}(\boldsymbol{\theta})\right| \stackrel{p}{\rightarrow} 0 .
$$

We have the following bounds:

$$
\begin{aligned}
& \max _{\theta \in N_{n}(\varepsilon)} \frac{1}{n}\left|h_{p, p}(\boldsymbol{\theta})-E h_{p, p}(\boldsymbol{\theta})\right| \\
= & \max _{\theta \in N_{n}(\varepsilon)} \frac{1}{n}\left|\sum_{i=1}^{n} x_{i p} x_{i p} e^{\boldsymbol{x}_{i}^{\top} \boldsymbol{a}}\left(\log \left(Z_{i}\right)-E \log \left(Z_{i}\right)\right)\right| \\
\leq & \max _{\theta \in N_{n}(\varepsilon)} \max _{\boldsymbol{x} \in K_{x}, \boldsymbol{y} \in K_{y}} C \sum_{i=1}^{n}\left|\frac{\log \left(Z_{i}\right)-E \log \left(Z_{i}\right)}{n}\right| \\
= & \max _{\theta \in N_{n}(\varepsilon)} \max _{\boldsymbol{x} \in K_{x}, \boldsymbol{y} \in K_{y}} C G_{n}
\end{aligned}
$$

From the law of large numbers and standard arguments, we can get $G_{n} \rightarrow 0$ in probability as $n \rightarrow \infty$. It remains to show, we will show

$$
\begin{aligned}
& \max _{\theta \in N_{n}(\varepsilon)}\left|\frac{1}{n}\left(f_{p, p}(\boldsymbol{\theta})-f_{p, p}\left(\boldsymbol{\theta}_{0}\right)\right)\right| \\
= & \max _{\theta \in N_{n}(\varepsilon)}\left|\frac{1}{n} \sum_{i=1}^{n} x_{i p} x_{i p}\left(e^{2 \boldsymbol{x}_{i}^{\top} \boldsymbol{a}} \psi_{1}\left(e^{\boldsymbol{x}_{i}^{\top} \boldsymbol{a}}\right)-e^{2 \boldsymbol{x}_{i}^{\top} \boldsymbol{a}_{0}} \psi_{1}\left(e^{\boldsymbol{x}_{i}^{\top} \boldsymbol{a}_{0}}\right)\right)\right| \\
\leq & \max _{\theta \in N_{n}(\varepsilon)} \frac{1}{n} \sum_{i=1}^{n}\left|x_{i p} x_{i p}\right| \cdot\left|e^{2 \boldsymbol{x}_{i}^{\top} \boldsymbol{a}_{1}} \psi_{1}\left(e^{\boldsymbol{x}_{i}^{\top} \boldsymbol{a}}\right)-e^{2 \boldsymbol{x}_{i}^{\top} \boldsymbol{a}_{0}} \psi_{1}\left(e^{\boldsymbol{x}_{i}^{\top} \boldsymbol{a}_{0}}\right)\right| \\
\leq & \frac{C}{n} \sum_{i=1}^{n}\left|x_{i p} x_{i p}\right| \max _{\theta \in N_{n}(\varepsilon)} \max _{\boldsymbol{x} \in K_{x}, \boldsymbol{y} \in K_{y}}\left|e^{2 \boldsymbol{x}^{\top} \boldsymbol{a}} \psi_{1}\left(e^{\boldsymbol{x}^{\top} \boldsymbol{a}}\right)-e^{2 \boldsymbol{x}^{\top} \boldsymbol{a}_{0}} \psi_{1}\left(e^{\boldsymbol{x}^{\top} \boldsymbol{a}_{0}}\right)\right| \\
\leq & C_{1} \max _{\theta \in N_{n}(\varepsilon)} \max _{\boldsymbol{x} \in K_{x}, \boldsymbol{y} \in K_{y}}\left|e^{2 \boldsymbol{x}^{\top} \boldsymbol{a}} \psi_{1}\left(e^{\boldsymbol{x}^{\top} \boldsymbol{a}}\right)-e^{2 \boldsymbol{x}^{\top} \boldsymbol{a}_{0}} \psi_{1}\left(e^{\boldsymbol{x}^{\top} \boldsymbol{a}_{0}}\right)\right| \\
= & : C_{G_{2 n}}
\end{aligned}
$$

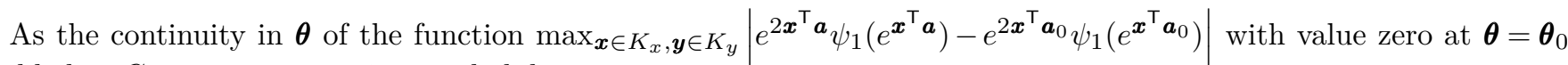
yield that $G_{2 n}$ converges to 0 in probability as $n \rightarrow \infty$

Theorem 2: Under the assumptions (A1)-(A3), the asymptotic normality of $\widehat{\boldsymbol{\theta}}$ can be obtained as the following

$$
\boldsymbol{F}_{n}^{\boldsymbol{\top} / 2} \cdot\left(\widehat{\boldsymbol{\theta}}-\boldsymbol{\theta}_{0}\right) \stackrel{D}{\longrightarrow} N_{P}\left(\mathbf{0}, \boldsymbol{I}_{p+q}\right)
$$


Proof Theorem 2: With the mean value theorem, we have

$$
\boldsymbol{s}_{n}\left(\widehat{\boldsymbol{\theta}}-\boldsymbol{\theta}_{0}\right)=\boldsymbol{F}_{n}\left(\boldsymbol{\theta}_{0}+\tau\left(\widehat{\boldsymbol{\theta}}-\boldsymbol{\theta}_{0}\right)\right) \cdot\left(\widehat{\boldsymbol{\theta}}-\boldsymbol{\theta}_{0}\right)
$$

for $0<\tau<1$ and $s_{n}(\widehat{\boldsymbol{\theta}})=0$. By pre-multiplying $\boldsymbol{F}_{n}^{\boldsymbol{\top} / 2}$ and integrating with respect to $\tau$ on [0,1], we have

$$
\boldsymbol{F}_{n}^{-1 / 2} \boldsymbol{s}_{n}=\left[\int_{0}^{1} \boldsymbol{V}_{n}\left(\boldsymbol{\theta}_{0}+\tau\left(\widehat{\boldsymbol{\theta}}-\boldsymbol{\theta}_{0}\right)\right) d \tau\right] \cdot \boldsymbol{F}_{n}^{\mathbf{\top} / 2}\left(\widehat{\boldsymbol{\theta}}-\boldsymbol{\theta}_{0}\right)
$$

Meanwhile, Lemma 5 implies that

$$
\int_{0}^{1} \boldsymbol{V}_{n}\left(\boldsymbol{\theta}_{0}+\tau\left(\widehat{\boldsymbol{\theta}}-\boldsymbol{\theta}_{0}\right)\right) d \tau \stackrel{p}{\rightarrow} \boldsymbol{I}_{p+q}
$$

By using Lemma 4 and the continuous mapping theorem [28], the asymptotic normality of $\widehat{\boldsymbol{\theta}}$ can be prove.

\section{Simulation STUdY}

In this section, we will provide some simulation experiments to illustrate our asymptotic theory and stability results.

\section{A. Fisher's scoring method}

The FS method is an efficient iterative algorithm for attempting to find the roots of a function $\boldsymbol{s}_{n}(\boldsymbol{\theta})$ by choosing a

starting value $\widehat{\boldsymbol{\theta}}_{(0)}$. The method for the score function is finding an iterative solution to the likelihood equations. As the modification of the NR method, the FS algorithm is an iterative method for finding the roots of a differentiable function that generates a sequence of estimates which usually come increasingly close to the optimal solution. The iteration is

$$
\widehat{\boldsymbol{\theta}}_{(j+1)}=\widehat{\boldsymbol{\theta}}_{(j)}+\boldsymbol{F}_{n}^{-1}\left(\widehat{\boldsymbol{\theta}}_{(j)}\right) \boldsymbol{s}_{n}\left(\widehat{\boldsymbol{\theta}}_{(j)}\right),
$$

which is the $j$ th iteration of the FS algorithm based on the observed Fisher information (OFI) matrix for estimating the parameters in the $G a$ GLM.

\section{B. Results for Asymptotic theory}

The numerical simulation based on Theorem 2 was conducted for the verification of the asymptotic properties of MLE of $G a \mathrm{GLM}$. To this end, the coefficients of $G a \mathrm{GLM}$ were estimated from various data sets independently generated by the same system. Then, the distributions of the estimated coefficients were compared with the normal distributions. Such experiments were repeated for different numbers of measurements $n$ sized as $\{50,100,200,500,1000,2000\}$. In this way, the relation between the convergence and $n$ can be investigated.

A simple model with intercept and covariate $1, x_{1}, x_{2}, y_{1}$ and $y_{2}$ were considered for the linear predictors $\boldsymbol{\eta}_{i}(\boldsymbol{\theta})^{\prime} s$, i.e. $\eta_{\alpha i}(\boldsymbol{\theta})=a_{0}+a_{1} x_{i 1}+a_{2} x_{i 2}$ and $\eta_{\beta i}(\boldsymbol{\theta})=b_{1} y_{i 1}+b_{2} y_{i 2}$ for $i=1, \ldots, n$. The values of the covariate $x_{1}, x_{2}, y_{1}$ and $y_{2}$ were chosen equally spaced between -1 and 1 . Further, for distinguishing the effects of different size parameters on the results, we examined set $a_{0}=-1, a_{1}=1, a_{2}=3, b_{1}=1$ and $b_{2}=3$. Since we are also interested in the case when $G a$ GLM does not satisfactorily fit the count regression data. For each combination of sample size $n$, setting, we simulated 100 samples of responses $Z_{i}$ 's, i.e. $Z_{i} \sim G a\left(\exp \left(a_{0}+a_{1} x_{i 1}+a_{2} x_{i 2}\right), \exp \left(b_{1} y_{i 1}+b_{2} y_{i 2}\right)\right)$ for $i=1, \ldots, n$.

We computed the average estimate, the estimated mean squared error (MSE), the mean absolute error (MAE) and the p-value [30] for univariate and multivariate normality test of the MLE $\hat{a}_{0}, \hat{a}_{1}, \hat{a}_{2}, \hat{b}_{1}$ and $\hat{b}_{2}$ respectively in 100 replications for each considered case, shown in table I. Simulation results reveal that the average estimate of each parameter close to the true value roughly as the sample size $n$ increases. With $n$ increase, the truncation error in the iterative process affects the estimation accuracy. The MSE and MAE decrease strictly as the number of samples increasing, demonstrate similar patterns.

We also test the normality of each parameter with estimating result by 100 replications. Due to the same range of randomly generated samples, when the number of samples is limited, i.e. $n=50,100$ and 200, the estimation of the smaller parameters will be affected by the bigger parameters. As the number of samples increases, when the number of samples reaches $n=2000$, the estimated value tends to be stable and presents a normal distribution.

A normal quantile-quantile (QQ) plots for the empirical distribution of multi-normal components are illustrated in figure 1. When the sample size is $n=50$ and 100, there are more outliers in the multivariate normal QQ plots. When the sample size increases to $n=200$ and 500, the QQ plots tend to be stable, when $n=1000$ and 2000, the QQ plots are normally distributed. 
TABLE I

Average estimate, standard deviation, estimated MSE, MAE and P-VAlue of $\hat{a}_{0}, \hat{a}_{1}, \hat{a}_{2}, \hat{b}_{1}$ And $\hat{b}_{2}$ FOr a GaGLM model on the BASIS OF 100 REPLICATIONS.

\begin{tabular}{|c|c|c|c|c|c|c|c|}
\hline Parameter & True value & $\mathrm{n}$ & Mean & MSE & MAE & $\mathrm{p}$-value & Normality test \\
\hline \multirow{6}{*}{$a_{0}$} & \multirow{6}{*}{-1} & 50 & -0.9868 & 0.2660 & 0.2158 & 0.0123 & $\mathrm{NO}$ \\
\hline & & 100 & -0.9798 & 0.1717 & 0.1344 & 0.2690 & YES \\
\hline & & 200 & -0.9972 & 0.1139 & 0.0902 & 0.9717 & YES \\
\hline & & 500 & -0.9975 & 0.0744 & 0.0577 & 0.0141 & $\mathrm{NO}$ \\
\hline & & 1000 & -0.9969 & 0.0507 & 0.0411 & 0.0064 & $\mathrm{NO}$ \\
\hline & & 2000 & -1.0011 & 0.0316 & 0.0249 & 0.8457 & YES \\
\hline \multirow{6}{*}{$a_{1}$} & \multirow{6}{*}{1} & 50 & 0.9561 & 0.4433 & 0.3659 & 0.7720 & YES \\
\hline & & 100 & 0.9446 & 0.2622 & 0.2095 & 0.6501 & YES \\
\hline & & 200 & 1.0040 & 0.1716 & 0.1346 & 0.0473 & $\mathrm{NO}$ \\
\hline & & 500 & 0.9905 & 0.1354 & 0.1110 & 0.1552 & YES \\
\hline & & 1000 & 1.0114 & 0.0844 & 0.0672 & 0.3956 & YES \\
\hline & & 2000 & 1.0009 & 0.0635 & 0.0488 & 0.3657 & YES \\
\hline \multirow{6}{*}{$a_{2}$} & \multirow{6}{*}{3} & 50 & 2.9553 & 0.4785 & 0.3861 & 0.8913 & YES \\
\hline & & 100 & 2.9721 & 0.2598 & 0.2129 & 0.1981 & YES \\
\hline & & 200 & 3.0030 & 0.1837 & 0.1367 & 0.7403 & YES \\
\hline & & 500 & 2.9869 & 0.1232 & 0.0997 & 0.5261 & YES \\
\hline & & 1000 & 2.9869 & 0.0888 & 0.0689 & 0.7357 & YES \\
\hline & & 2000 & 2.9785 & 0.0634 & 0.0520 & 0.0964 & YES \\
\hline \multirow{6}{*}{$b_{1}$} & \multirow{6}{*}{1} & 50 & 1.0854 & 0.5467 & 0.4576 & 0.0487 & $\mathrm{NO}$ \\
\hline & & 100 & 1.0024 & 0.3859 & 0.3081 & 0.0176 & $\mathrm{NO}$ \\
\hline & & 200 & 1.0014 & 0.2281 & 0.1828 & 0.3867 & YES \\
\hline & & 500 & 0.9973 & 0.1647 & 0.1297 & 0.6182 & YES \\
\hline & & 1000 & 0.9922 & 0.1122 & 0.0911 & 0.0717 & YES \\
\hline & & 2000 & 0.9852 & 0.0748 & 0.0586 & 0.8467 & YES \\
\hline \multirow{6}{*}{$b_{2}$} & \multirow{6}{*}{3} & 50 & 3.0246 & 0.5903 & 0.4588 & 0.7750 & YES \\
\hline & & 100 & 3.0225 & 0.3148 & 0.2526 & 0.2990 & YES \\
\hline & & 200 & 3.0388 & 0.2618 & 0.2072 & 0.5040 & YES \\
\hline & & 500 & 2.9603 & 0.1706 & 0.1345 & 0.2272 & YES \\
\hline & & 1000 & 2.9592 & 0.1230 & 0.1006 & 0.4994 & YES \\
\hline & & 2000 & 2.9848 & 0.0933 & 0.0715 & 0.4488 & YES \\
\hline
\end{tabular}

Figure 2 illustrates the convergence of parameters estimation by different size of samples. With the increase of samples, the mean value of each estimated parameter gradually approaches the real value, and the fluctuation range gradually decreases, that is, the variance decreases. Therefore, it indicates that the parameter estimation value converges to the actual value, and the maximum likelihood estimate is consistent. Among them, it can be seen from the figure that due to the difference in parameter size, the bigger parameter is easier to converge to the real value, which has the significance of estimation.

\section{Conclusion}

GaGLM is now commonly used for investigating positive real data. In this paper, similar to the asymptotic theory of MLE of natural exponential distribution family-based GLM, we establish the consistency and asymptotic normality of MLE of GaGLM by proving the Lyapunov condition. We also discuss the range of the logarithmic $k$-order expectation $E(\log Z)^{k}$ as $k=1,2, \ldots, 6$ when $Z$ obey the Gamma distribution. Moreover, the simulation study illustrates that the normal approximation is satisfactory for moderate and large sample sizes. Finally, with the established asymptotic theory, we can further benefit interval estimates, hypothesis tests and stochastic control design in a theoretical basis.

\section{REFERENCES}

[1] J. A. Nelder and J. W. Hardin, "Generalized Linear Models," J. Royal Statistical Society Series A, vol. 135, no. 3, pp. 370-382, Apr. 1972 .

[2] E. W. Stacy, "Generalization of gamma distribution," Ann. Mathe. Stat., vol. 33, no. 3, pp. 1187-1192, 1962.

[3] S. K. Agarwal and S. L. Kalla, "A generalized gamma distribution and its application in reliability," Commu. Stat. Theo. Meth., vol. 25, no. 1, pp. 201-210, 1996.

[4] A. Mahmoudi, R. A. Belaghi, S. Mandal, "A comparison of preliminary test, Stein-type and penalty estimators in gamma regression model," Statistics in medicine, vol. 90, no. 17, pp. 3051-3079, Nov. 2020.

[5] S. Mandal, R. Arabi Belaghi, A. Mahmoudi and M. Aminnejad, "Stein-type shrinkage estimators in gamma regression model with application to prostate cancer data," Statistics in medicine, vol. 38, no. 22, pp. 4310-4322, Sep. 2019.

[6] S. Ecker, V. Pancaldi, D. Rico, and A. Valencia, "Higher gene expression variability in the more aggressive subtype of chronic lymphocytic leukemia," bioRxiv, 005637, 2014.

[7] K. Summun, N. Mamode Khan, Z. Jannoo, Y. Sunecher and I. Veerasamy, "An assessment of the determinants of Mauritian automobile insurance claims using negative binomial and gamma regression models," J. Statist. Manage. Syst., vol. 21, no. 5, pp. 725-740, Aug. 2018.

[8] G. Agogo, "A zero-augmented generalized gamma regression calibration to adjust for covariate measurement error: A case of an episodically consumed dietary intake," Biometrical J., vol. 59, no. 1, pp. 94-109, Jan. 2017.

[9] E. I. George, "The variable selection problem," J. Amer. Statist. Assoc., vol. 95, no. 452, pp. 1304-1308, Dec. 2000. 

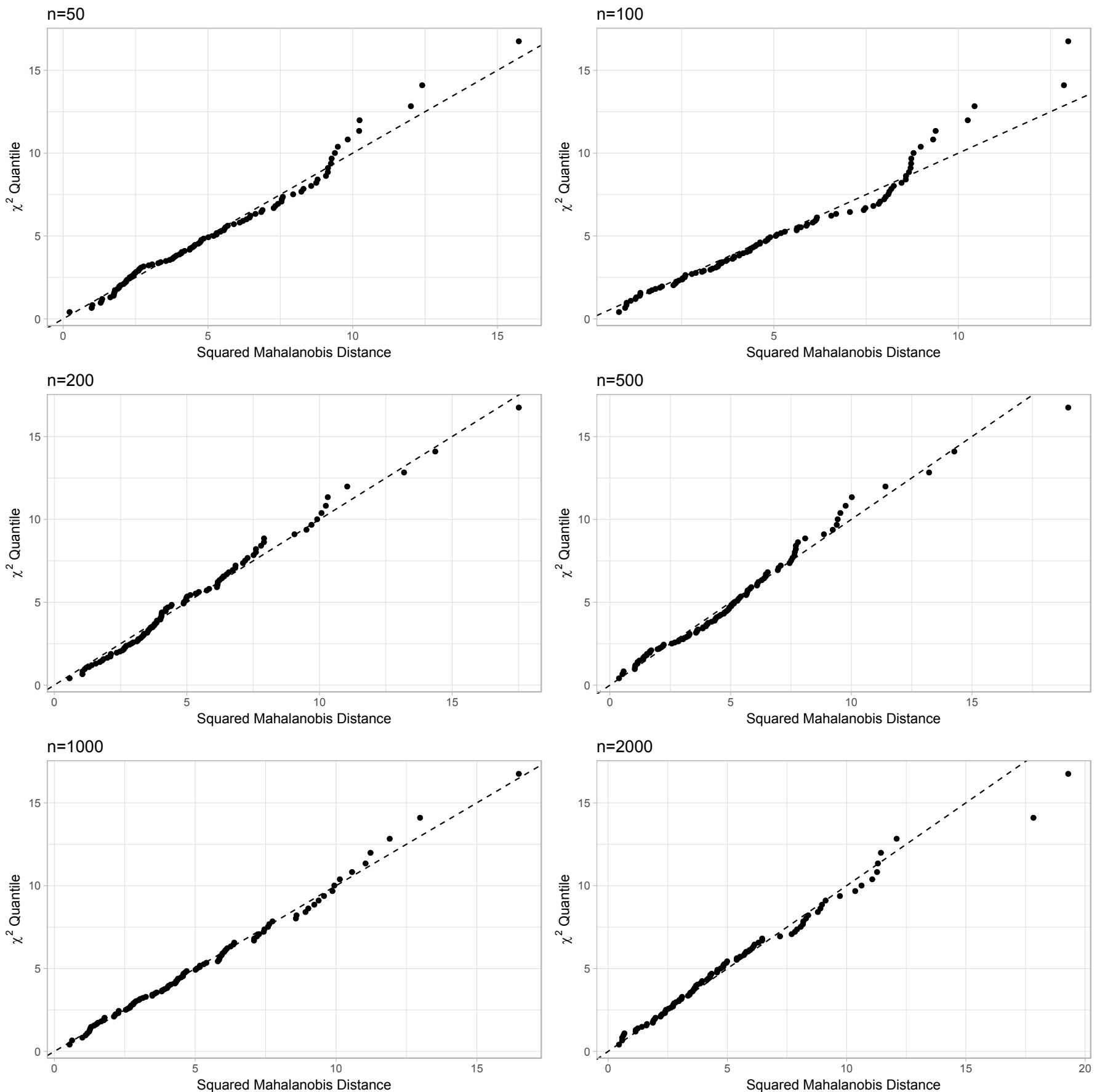

Fig. 1. Normal QQ-plots of centered and normalized ML estimators for a GaGLM model based on 100 replications.

[10] P. Li, D. Paul, R. Narasimhan, J. Cioffi, "On the distribution of SINR for the MMSE MIMO receiver and performance analysis," IEEE Trans. Inf. Theory, vol. 52, no. 1, pp. 271-286, Jan. 2006.

[11] M. Kazeminia and M. Mehrjoo, "A New Method for Maximum Likelihood Parameter Estimation of Gamma-Gamma Distribution," J. Lightwave Technology, vol. 31, no. 9, pp. 1347-1353, Mar. 2013.

[12] T. J. Ypma, "Historical Development of the NewtonRaphson Method," SIAM Rev., vol. 37, no. 4, pp. 531-551, 1995.

[13] J. Guo, L. Y. Wang, G. Yin, Y. Zhao, and J. F. Zhang, "Asymptotically efficient identification of FIR systems with quantized observations and general quantized inputs," Automatica, vol. 57, pp. 113-122, Jul. 2015.

[14] L. Fahrmeir and H. Kaufmann, "Consistency and asymptotic normality of the maximum likelihood estimator in generalized linear models," Ann. Statist., vol. 13, no. 1, pp. 342-368, 1985.

[15] R. Ash and C. Doleans-Dade, "Probability and Measure Theory," Academic Press, 2000.

[16] A. Leucht, "Degenerate U- and V-statistics under weak dependence: Asymptotic theory and bootstrap consistency," Bernoulli, vol. 18, no. 2, pp. 552-585, 2012.

[17] Y. Zeng, H. Li, N. M. Schweppe, R. S. Hawley and W. D. Gilliland, "Statistical analysis of nondisjunction assays in drosophila," Genetics, vol. 186, no. 2, pp. 505-U104, 2010.

[18] P. McCullagh and J. Nelder, "Generalized linear models," Chapman Hall., 1989. 

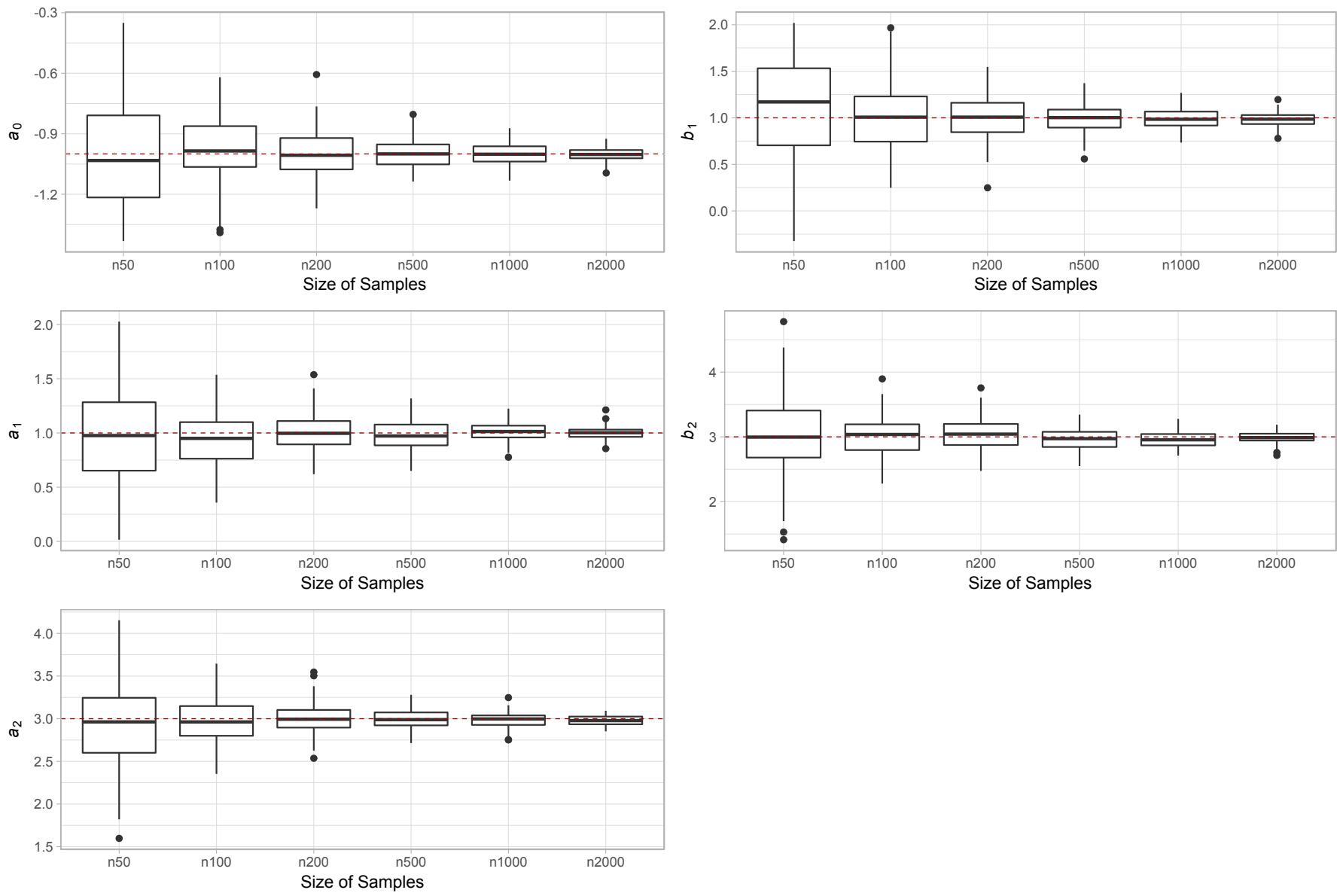

Fig. 2. Mean and variance of the regression coefficients corresponding to each of the sample size.

[19] R. I. Jennrich and P. F. Sampson, "Newton-Raphson and related algorithms for maximum likelihood variance component estimation," Technometrics, vol. 18, no. 1, pp. 11-17, 1976.

[20] H. Cramer, "Mathematical Methods of Statistics," Princeton University Press, Princeton, 1946.

[21] K. Liang, "The asymptotic efficiency of conditional likelihood methods," Biometrika, vol. 71, no. 2, pp. 305-313, 1984.

$[22]$ T. Y Hwang and P. H. Huang, "On New Moment Estimation of Parameters of the Gamma Distribution Using its Characterization," Ann. Inst. Stat. Math., vol. 54, no. 4, pp. 840-847, Dec. 2002.

[23] G. E. Andrews, R. Askey, and R. Roy, "Special functions," Cambridge Univ. Press, 1999.

[24] K. V. Mardia, J. T. Kent and J. M. Bibby, "Multivariate analysis," London: Academic Press, 1979.

[25] E. L. Lehmann and G. Casella, "Theory of point estimation," Springer Science \& Business Media., 2006.

[26] J. Hoffmann-Jorgensen, "Probability with a view toward statistics," New York: Chapman \& Hall., 1994.

[27] V. V. Petrov, "Limit theorems of probability theory: sequences of independent variables," Oxford: Clarendon Press., 1995.

[28] P. Billingsley, "Convergence of Probability Measures," John Wiley \& Sons, 2013.

[29] C. Czado, V. Erhardt and A. Min, "Zero-inflated generalized Poisson models with regression effects on the mean, dispersion and zero-inflation level applied to patent outsourcing rates," Statist. Mod., vol. 7, no. 2, pp. 125-153, Jul. 2007.

[30] N. Henze and B. Zirkler, "A class of invariant consistent tests for multivariate normality," Commun. Statist. Theory Methods, vol. 19, no. 10, pp. 3595-3617, 1990.

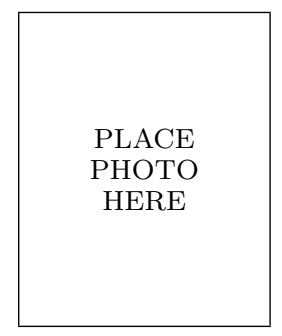

Benchao Wang received the M.En. degree in control theory and control engineering from the Dalian University of Technology, Dalian, China, in 2012. He is a Ph. D. student with the Faculty of Electronic Information and Electrical Engineering, the Dalian University of Technology, Dalian, China, from 2013 to now.He is currently a teacher of Liaoning Police College, Dalian, China.

PLACE His current research interests include statistical modeling and stochastic control theory. 


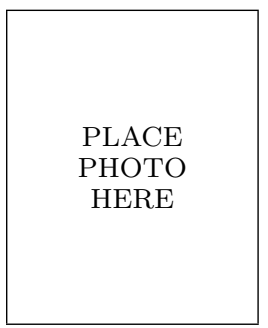

Hong Gu received the B.Sc. and M.Sc. degrees from the Shenyang University of Technology, Shenyang, China, in 1982 and 1984, respectively, and the Ph.D. degree from Zhejiang University, Hangzhou, China, in 1990.

Since 1996, he has been a Full Professor with the Dalian University of Technology, Dalian, China. His current research interests include machine learning, big data, and bioinformatics.

Pan Qin received the Ph.D. degree in information science from Kyushu University, Fukuoka, Japan, in 2008. He was a Research Fellow with the Faculty of Mathematics and the Institute of Mathematics for Industry, Kyushu University, from 2008 to 2013. He is currently an Associate Professor with the Faculty of Electronic Information and Electrical Engineering, Dalian University of Technology, Dalian, China.

His current research interests include statistical modeling and stochastic control theory. 